\title{
Temperature Dependent Molecular Dynamic Simulation of Friction
}

\author{
R. A. Dias, M. Rapini, B. V. Costa, \\ Laboratório de Simulação, Departamento de Física, ICEX, UFMG 30123-970, Belo Horizonte, MG, Brazil \\ and P. Z. Coura \\ Departamento de Física, ICE, UFJF, Juiz de Fora, MG, Brazil
}

Received on 28 September, 2005

\begin{abstract}
In this work we present a molecular dynamics simulation of a $F F M$ experiment. The tip-sample interaction is studied by varying the normal force in the tip and the temperature of the surface. The friction force, $c A$, at zero load and the friction coefficient, $\mu$, were obtained. Our results strongly support the idea that the effective contact area, $A$, decreases with increasing temperature and the friction coefficient presents a clear signature of the premelting process of the surface.
\end{abstract}

Keywords: Nanometric friction; Molecular dynamic; Surface premelting

\section{INTRODUCTION}

Friction is one of the oldest phenomenon studied in natural sciences. In a macroscopic scale it is known that the friction force between surfaces satisfies the following rules: (1) The friction is independent of contact area between surfaces; (2) It is proportional to the normal force applied between surfaces and (3) The kinetic friction force is independent of relative speed between surfaces[1]. Considering that friction is the result of many microscopic interactions between the building atoms at the surfaces, it must depend on factors as roughness, temperature and the energy dissipation mechanism at the surfaces. Therefore, to understand its macroscopic behavior it is necessary to understand in details the dynamics of interaction between atoms in the surfaces in contact. In 1987, C. M. Mat et al[2] have used, for the first time, the Friction Force Microscope (FFM) to investigate friction in nano-scale. That kind of microscope allows the experimentalist to produce essentially a single contact between a sharp tip, of atomic dimensions, and the surface. [3, 4].

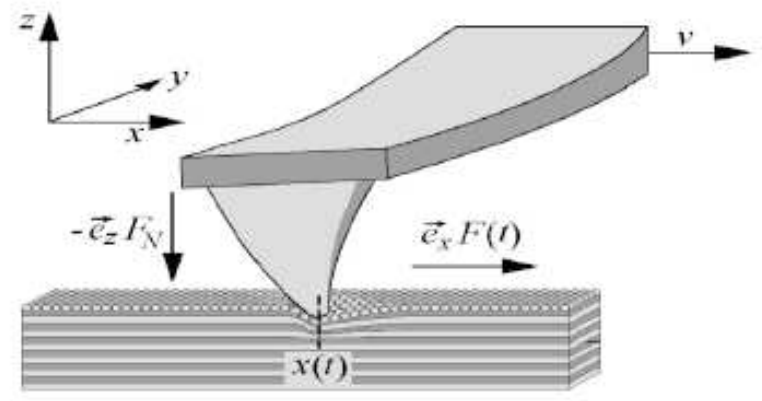

FIG. 1: Schematic view of a FFM apparatus. [5]

Its basic principle is shown in Fig. 1. In this apparatus the tip stays in touch with the surface while it moves at constant velocity, $v$, parallel to the surface. The resulting force in the cantilever makes a torsion on it. This torsion can be observed by optical techniques. One of the more striking effects ob- served is the stick-slip phenomenon: The friction force plotted as a function of time exhibits a sawtooth behavior. [6, 7].

Analytically, the motion of the tip can be modelled as follows. Forces in the tip are a sum of two terms: An interaction force between the tip and the surface due to the periodic atomic arrangement of the lattice and a force due to the cantilever. Some authors by using this approach were able to reproduce several features of the friction at a nanoscopic scale $[8,9]$. In this work we use molecular dynamics (MD) simulation to study the friction phenomenon at the atomic scale. In Fig. 2 we show a schematic view of the model we have used in our simulation to reproduce the FFM mechanism (Figure 1). The tip is represented by a single particle that interacts with the surface through a convenient potential. The springs represent the mechanism we have used to vary the normal force ( $z$ direction) and to measure the lateral force ( $x$ and $y$ directions). By measuring both forces it is possible to study the friction force behavior under several circumstances.

In a recent work Resende and Costa [10] using molecular dynamic simulation have studied the migration of an individual atom on the surface of a 12-6 Lennard-Jones bcc crystal. They argued that an observed anomaly occurring in the diffusion constant could be the signature of a pre-melting process. The migration of an ad-atom at the surface may occurs by three mechanisms. At low temperature the adsorbed particle can move through channels on the surface since thermal motion of atoms at the surface have low amplitude. Once temperature rises one reaches an intermediate state. The surface starts to melt so that the channels are closed and ad-atoms are stuck in the vicinity of a surface atom. The situation persists until the ad-atom is thermally activated and random-walk diffusion occurs. In summary, the diffusion constant should present a minimum at the intermediate region. Under the point of view of friction we may ask what is the effect of this phenomenon over friction. For two macroscopic sliding surfaces we may not expect to distinguish the first two process since the contact area is large compared to interatomic distance. However as temperature rises the surface is lubricated by melted atoms, we may expect a smaller friction coefficient. The situation is quite different for a small tip in contact with the surface. 


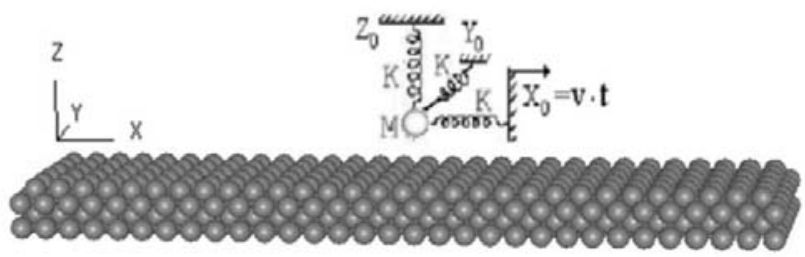

FIG. 2: Schematic view of our Friction Force apparatus.

In the following we describe a series of molecular dynamics computer simulation of the interaction of a small tip with a surface. Computer simulations give us a very convenient instrument to study tribological processes. They allow controlled "numerical experiments" where the geometry, sliding conditions and interactions between atoms can be varied at will to explore their effect on friction, lubrication, wear and to reach unaccessible regions for experimentalists. Unlike laboratory experiments, computer simulations enable us to follow and analyze the full dynamics of all atoms. A wide range of potentials have been employed in numerical studies of tribology. For example, simulations of metals frequently use the embedded atom method, while studies of hydrocarbons use potentials that include bond-stretching and so on. We will not concentrate ourselves in any specific material rather in general aspects of the problem under consideration. Due to the gen- eral character of our study we content ourselves by using the Lennard-Jones $(6-12)$ potential which is commonly used in studies of general behavior. We will consider that the interaction of the tip with the surface does not disturb very much the electronic structure of the system. This consideration means that we do not have to deal with the quantum aspects of the problem. This simplification let us save a lot of computer time. If, for one side, we lose details of the considered phenomenon, we gain in that we can study true dynamical and temperature dependent models.

This work is organized as follows. In the section II we introduce general aspects of the numerical method used, in the section III we present our results and in section IV we discuss and present some conclusions.

\section{SIMULATION BACKGROUND}

Our simulation is carried out by using molecular dynamics (MD) simulation. A schematic view of the simulation arrangement we have used is shown in Fig. 2. Three springs of elastic constants $k_{x}, k_{y}$ and $k_{z}$ are attached to the particle $M$ that represents a tip point. This arrangement allow us to measure normal $\left(F_{z}\right)$ and parallel $\left(F_{x}, F_{y}\right)$ forces on $M$. The surface is represented by an arrangement of particles which interact with each other and with the mass $M$ through a truncated Lennard-Jones $(6-12)(\mathrm{LJ})$ potential

$$
\Phi_{i, j}\left(r_{i, j}\right)= \begin{cases}\phi_{i, j}\left(r_{i, j}\right)-\phi_{i, j}\left(r_{c}\right)-\left(r_{i, j}-r_{c}\right)\left(\frac{\partial \phi_{i, j}\left(r_{i, j}\right)}{\partial r_{i, j}}\right)_{r_{i, j}=r_{c}} & \text { if } r_{i, j}<r_{c} \\ 0 & \text { if } r_{i, j}>r_{c}\end{cases}
$$

where $\phi_{i, j}\left(r_{i, j}\right)$ is the complete $\mathrm{LJ}$ potential,

$$
\phi_{i, j}\left(r_{i, j}\right)=4 \varepsilon_{i, j}\left[\left(\frac{\sigma_{i, j}}{r_{i, j}}\right)^{12}-\left(\frac{\sigma_{i, j}}{r_{i, j}}\right)^{6}\right] .
$$

The indexes $i$ and $j$ stands for position vectors $\overrightarrow{r_{i}}$ and $\overrightarrow{r_{j}}$ respectively, and $1 \leq i \leq N$, where $N$ is the total number of particles and $r_{i, j}=\left|r_{j}-r_{i}\right|$. A cutoff, $r_{c}$, is introduced in the potential in order to accelerate the simulation. If the force on a particle is found by summing contributions from all particles acting upon it, then this truncation limits the computation time to an amount proportional to the total number of particles $N$. Of course, this truncation introduces discontinuities both in the potential and the force. To smooth these discontinuities we introduce the constant term $\phi\left(r_{c}\right)$. Another term $\left(\partial \phi_{i, j}\left(r_{i, j}\right) / \partial r_{i, j}\right)_{r_{i, j}=r_{c}}$ is introduced to remove the force discontinuity. Particles in the simulation move according to Newton's law of motion, which generates a set of $3 N$ coupled equations of motion which are solved by increasing forward in time the physical state of the system in small time steps of size $\delta t$. The resulting equations are solved by using Beeman's method of integration[11-14]. The system is arranged in 4 layers with free boundary conditions in all directions. The first layer is frozen in a regular arrangement as in the (001) surface of a Lennard-Jones bcc crystal in order to maintain the whole structure as flat as possible.

With the tip far away from the surface we thermalize the system at temperature $T$. After thermalization, the tip is pushed in a direction parallel to the surface at constant velocity $v_{p}$. For each simulation the distance between the spring and the surface is fixed at the start, so that we can control the perpendicular force on the tip. By measuring the size variation of the springs we can calculate the lateral, $F_{x}$, and the perpendicular force, $F_{z}$, on the tip. The temperature, $T$, of the surface can be controlled by using a velocity renormalization scheme (See for example [15] and references therein). From the equipartition theorem we can write that

$$
\left\langle v^{2}\right\rangle=3 \frac{k_{B}}{m} T .
$$

By controlling the value of $\left\langle v^{2}\right\rangle$ we can reach a chosen temperature $T_{f}$. An appropriated way to do that is by successive 


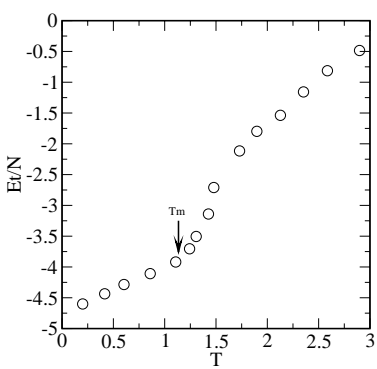

FIG. 3: Energy as a function of temperature. The melting temperature is estimated as the inflexion point, being around $T_{m} \approx 1.1 \varepsilon / k_{b}$

approximations. By multiplying each velocity by a factor $\alpha_{0}$ defined as

$$
\alpha_{0}=\sqrt{\frac{m}{3 k_{B}} \frac{\left\langle v^{2}\right\rangle_{0}}{T_{f}}},
$$

a first approximation to $T_{f}$ is done. By evolving in time the system we can create sequences, $T_{n},\{v\}_{n}$ and $\{\alpha\}_{n}$, such that after a finite number of time steps the temperature of the system converges to $T_{n} \approx T_{f}$. The friction coefficient is calculated as the quotient

$$
\mu \equiv \frac{d F_{x}}{d F_{z}}
$$

Before we start the simulation we have to have an estimative of the melting temperature, $T_{m}$, of the system. This is done by performing a preliminary simulation of the substrate. In Figure 3 we show the total energy per particle, $E$, as a function of temperature. The melting temperature is estimated as the inflection point of the curve. We find $T_{m} \approx 1.1$ in accordance with earlier calculations [10, 15-17]. The velocity, position and forces are stored at each time step for further analysis. We measure the time $t$, temperature $T$ and forces in units of $\sigma \sqrt{m / \varepsilon}, \varepsilon / k_{B}$ and $\varepsilon / \sigma$ respectively.

\section{RESULTS}

We have simulated the FFM system for several temperatures and initial distances of the tip to the substrate or equivalently, the normal force in the tip. In Fig. 4 we show a plot of our MD simulation results for the friction force as a function of normal force for several temperatures. The Amonton's Law of friction states that frictional forces are proportional to the normal force and independent of the contact area. This type of behavior was observed in some systems by many authors, who fitted $\left\langle F_{x}\right\rangle$ to a linear function of both load, $\left\langle F_{z}\right\rangle$, and contact area, $A$ :

$$
\left\langle F_{x}\right\rangle=\mu\left\langle F_{z}\right\rangle+c A
$$

Here $\mu$ is the friction coefficient and the second term $c A$ is interpreted as the friction force for zero normal force. In the
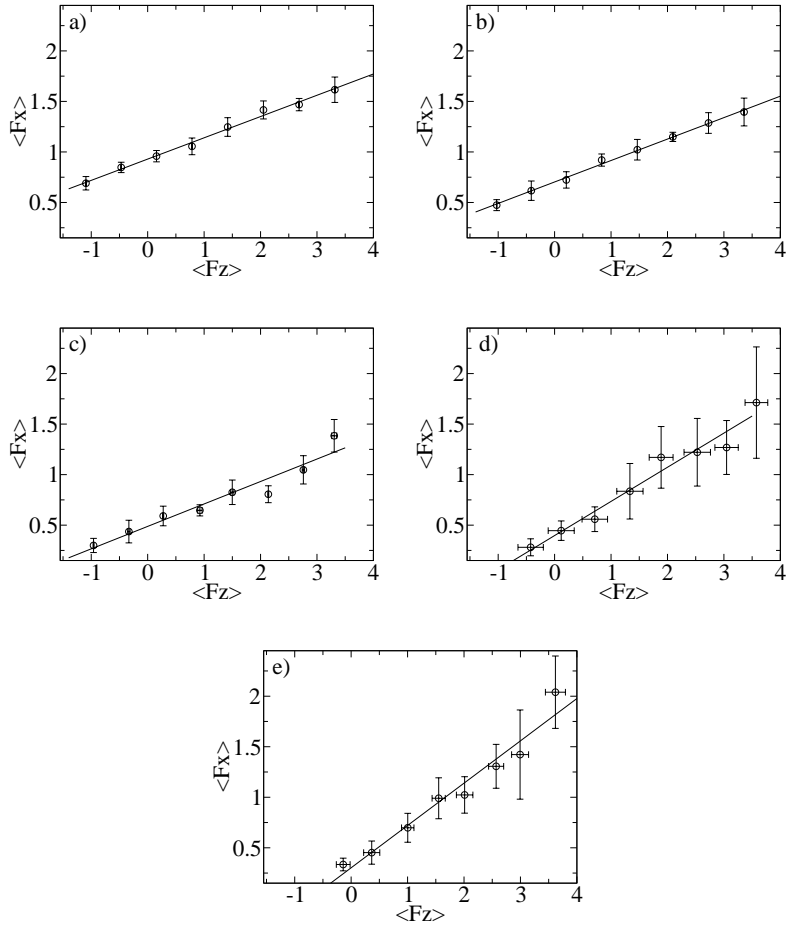

FIG. 4: The friction force, $\left\langle F_{x}\right\rangle$ as a function of normal force, $\left\langle F_{z}\right\rangle$ is shown for several temperatures. The $\left\langle F_{x}\right\rangle$ and $\left\langle F_{z}\right\rangle$ forces are measured in units of $\varepsilon / \sigma$. The figures, from $a$ to $e$ are for several different values of $T=0.25,0.44,067,085,1.05$ respectively. The circles are the MD results and the straight line correspond to an adjust.

following we present our results that strongly suggest that the Amonton's laws $[18,19]$ is violated when the friction force is considered as a function of temperature. In the Fig. 5(left) we show a plot of $c A$ as a function of temperature. We can see that when the temperature increases the contact area or adhesion forces decrease. This behavior can be related to the fact that at low temperature the atoms at the surface perform low amplitude jumps so that the number of collisions with the tip is low. In this case the effective contact area is high because the tip stays a long time close to the surface. However, when the temperature grows the number of high energy fluctuations of particles at the surface increases with a consequent increase in the number of high energy collisions with the tip, decreasing the effective contact area. We also observe that the friction coefficient (Shown in Fig. 5(right)) grows abruptly at $T \sim 0.7 \varepsilon / k_{b}$. This behavior can be related to the pre-melting of the surface. We show in fig. 6 a plot of the path of the tip over the surface for several temperatures and normal forces. As should be expected the paths are well defined for low temperatures becoming random as temperature grows. To understand what is going on, we have calculated the residence time of the tip in each site, defined as the time that the tip spends in the neighborhood of a specific site, i.e., its distance to some particular site being smaller than some reference distance $\delta$. With no loss of generality we choose $\delta=\sigma$, the lattice parameter. In Figs. 7 and 8 we show the histograms for 

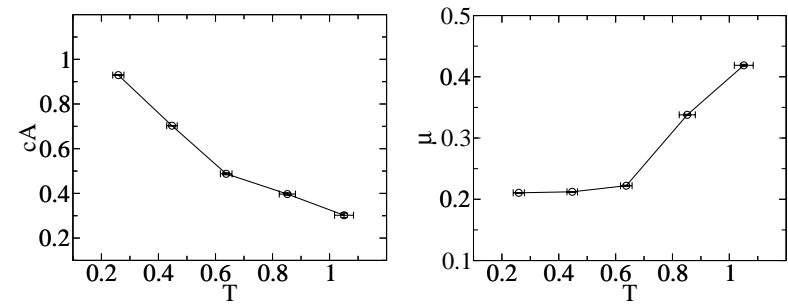

FIG. 5: Plot of $c A$ (left) and $\mu$ (right) as a function of Temperature. The line is only a guide to the eyes.
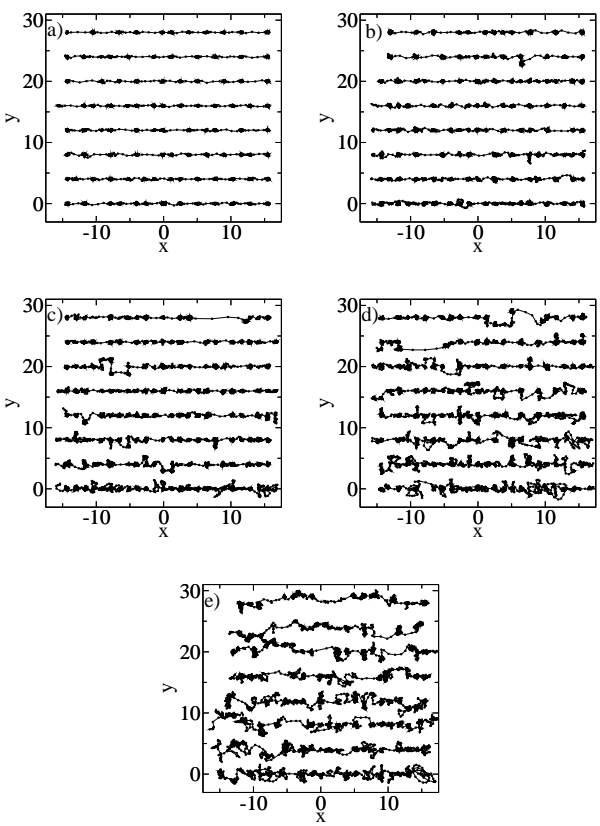

FIG. 6: Path of the tip over the surface (XY plane) for several temperatures and normal forces. From a) to e) we have $T=0.25,0.44,067,085,1.05$ respectively. The normal forces are defined in fig 4 . The plots are shown dislocated by a constant value in the $y$ direction as a matter of clarity.

normal forces $F_{z}=-1.09,-1.02,-0.95,-0.42,-0.14$ and $F_{z}=2.05,2.09,2.13,2.52,2.56$ respectively for several temperatures. For negative values of $F_{z}$ the residence time is well defined even at high temperatures having its average at $\delta t \approx 50$. The tip is immersed in the surface, so that, it can easily travel along channels on the surface of the crystal. In the Fig. 8 we show the histograms for positive values of $F_{z}$. The temperatures are the same as in Fig. 4. At low T they show a similar behavior as that for $F_{z}<0$. However, at higher temperatures, the residence time spreads out to the higher $t$ region. We interpret this as a closing of the channels discussed above due to thermic motion of the particles at the surface. When temperature increases the particle gets more energy, which is eventually enough to push it from any specific neighborhood.
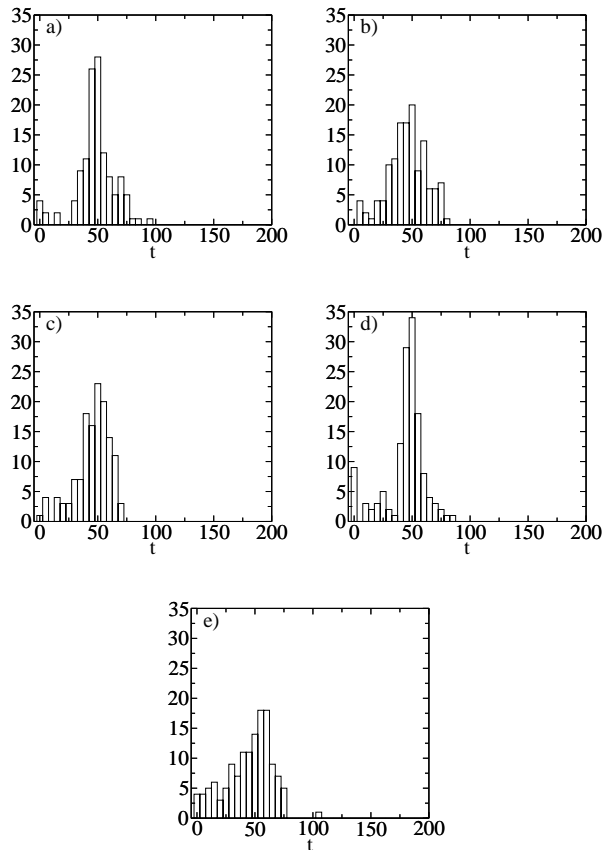

FIG. 7: Histogram for the residence time. The normal force is for $F_{z}=$ $-1.09,-1.02,-0.95,-0.42,-0.14$. Beam size is $t_{\text {beam }}=5$.
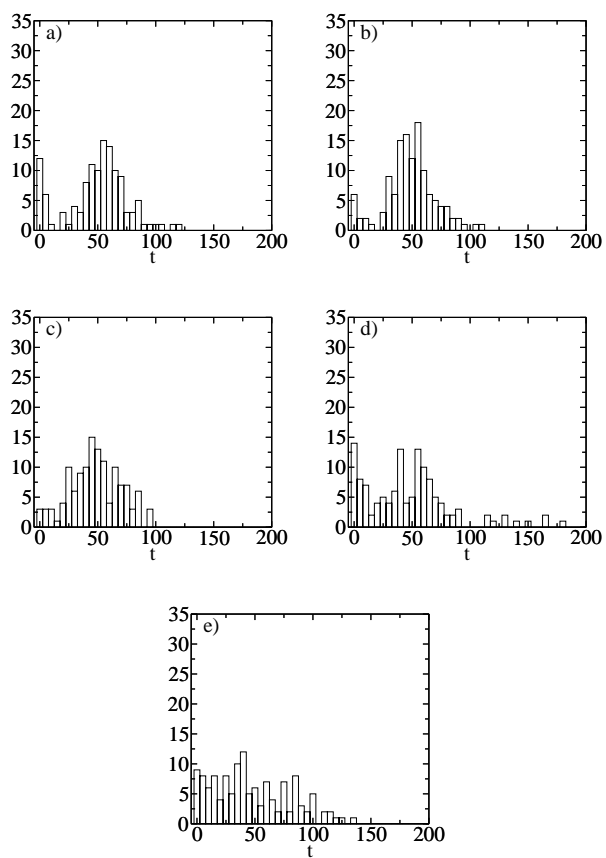

FIG. 8: Histogram for the residence time. The normal force is for $F_{z}=$ $2.05,2.09,2.13,2.52,2.56$. Beam size is $t_{\text {beam }}=5$. 


\section{CONCLUSION}

We have performed a molecular dynamics simulation of a FFM experiment. Our results were obtained by varying the normal force in the tip and the temperature of the surface. The behavior of the $c A$ term in the Amonton's law (eq. 6) and the friction coefficient were found to depend on the temperature. The $c A$ term which measures essentially the effective contact area, $A$, between the tip and the surface were found to decrease with increasing $T$. The friction coefficient presents a sudden jump which seems to be related to the premelting processes of the surface.

\section{Acknowledgments}

This work was partially supported by CNPq. We are grateful to B.A. Soares for suggestions and comments.
[1] E. Meyer, R. M. Overney, K. Dransfeld, and T. Gyalog, Nanoscience - Friction and Rheology on the Nanometer Scale, (World Scientific Publishing, Singapore, 1998).

[2] C. M. Mate, G. M. McClelland, R. Erlandsson, and S. Chiang, Phys. Rev. Lett. 59, 1942 (1987).

[3] S. Fujisawa, E. Kishi, Y. Sugawara, and S. Morita, Phys. Rev. B 51, 7849 (1995).

[4] R. Lüthi, E. Meyer, M. Bammerlin, L. Howald, H. Haefke, T. Lehmann, C. Loppacher, H.-J. Güntherodt, T. Gyalog, and H. Thomas, J. Vac. Sci. Technol. B 14, 1280 (1996).

[5] P. Reimann and M. Evstigneev, Description of atomic friction as forced Brownian motion New Journal of Physics, 7, 25 (2005).

[6] T. Gyalog and H. Thomas, Z. Phys. B 104, 669 (1997).

[7] A. Volmer and Th. Nattermann, Z. Phys. B 104, 363 (1997).

[8] E. Gnecco et al., Phys. Rev. Lett. 84, 1172, (2000).

[9] P. Reimann and M. Evstigneev Phys. Rev. Lett. 93, 230802
(2004)

[10] F. J. Resende and B. V. Costa, Phys. Rev. B 61, 12697 (2000).

[11] D. Bemman, J. Comput. Phys. 20, 130 (1976).

[12] H. J. C. Berendsen and W. F. Gunsteren Pratical Algorithms for Dynamic Simulations. Pag. 43-65.

[13] M. P. Allen and D. J. Tildesley, Computer Simulation of Liquids (Oxford Scince Publications, New York, 1992)

[14] D. C. Rapaport, The Art of Molecular Dynamic Simulation (Cambridge University Press, New York, 1997)

[15] P. Z. Coura, O. N. Mesquita and B. V. Costa, Phys. Rev. B 59, 3408 (1999).

[16] F. J. Resende and B. V. Costa, Surf. Sci. 481, 54 (2001).

[17] P. Z. Coura and B. V. Costa, Int. J. Mod. Phys. C 9, 857 (1998).

[18] J. Ringlein and M. O. Robbins, Am. J. Phys. 72, 884 (2004).

[19] D. Gourdon, N. Israelachvili, Phys. Rev. E 68, 021602, (2003). 